PHYSICAL REVIEW D 96, 059903(E) (2017)

\title{
Erratum: Transverse energy density fluctuations in heavy-ion \\ collisions in a Gaussian model \\ [Phys. Rev. D 85, 114030 (2012)]
}

Berndt Müller and Andreas Schäfer

(Received 3 September 2017; published 28 September 2017)

DOI: $10.1103 /$ PhysRevD.96.059903

We have recently learned that Eqs. $(20,22)$ of our article on initial-state transverse energy density fluctuations in relativistic heavy ion collisions (henceforth denoted as MS) contains algebraic errors. We here present the corrected expressions and revised figures. The correct equations are as follows:

$$
\begin{gathered}
M_{5}+M_{6}+M_{8}+M_{9}=\frac{g^{4}}{16} N^{2}\left(N^{2}-1\right)\left[D(\mathbf{z})^{2}-E(\mathbf{z})^{2}-F(\mathbf{z})^{2}\right]^{2}, \\
M_{1}=\frac{g^{4}}{4} N^{2}\left(N^{2}-1\right)^{2} D(0)^{4}+\frac{g^{4}}{2} N^{2}\left(N^{2}-1\right) D(0)^{2} K(\mathbf{z})+\frac{3 g^{4}}{16} N^{2}\left(N^{2}-1\right) K(\mathbf{z})^{2},
\end{gathered}
$$

where $\mathbf{z}=\mathbf{x}-\mathbf{y}$ and $K(\mathbf{z})$ is given by (25) in MS. Combining these equations, the correct expression for the transverse energy density fluctuations [Eq. (23) in MS] becomes

$$
\langle\varepsilon(\mathbf{x}) \varepsilon(\mathbf{y})\rangle-\langle\varepsilon(\mathbf{x})\rangle\langle\varepsilon(\mathbf{y})\rangle=\frac{g^{4}}{4} N^{2}\left(N^{2}-1\right)\left[2 D(0)^{2} K(\mathbf{z})+D(\mathbf{z})^{4}+K(\mathbf{z})\left(E^{2}(\mathbf{z})+F^{2}(\mathbf{z})\right)\right] .
$$

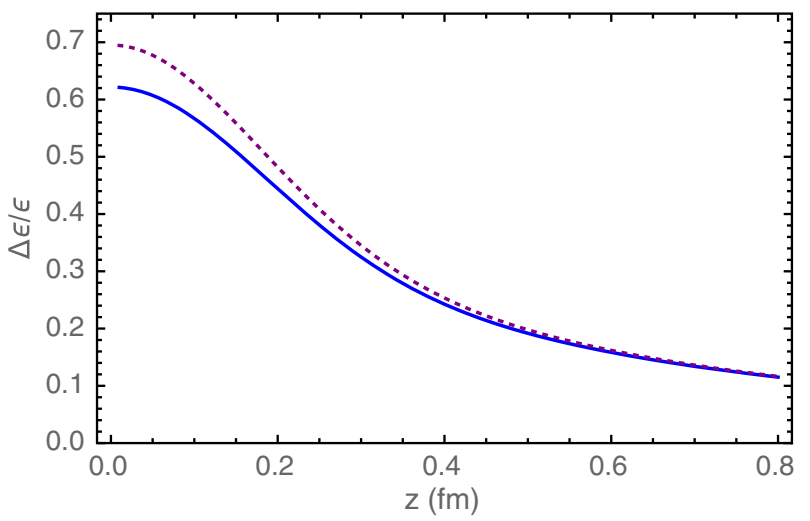

FIG. 1. The function $\Delta \varepsilon(z) / \varepsilon_{0}$ for the selected parameters (solid blue line). The incorrect result shown in Fig. 3 of our original publication is shown as a dashed red line for comparison.

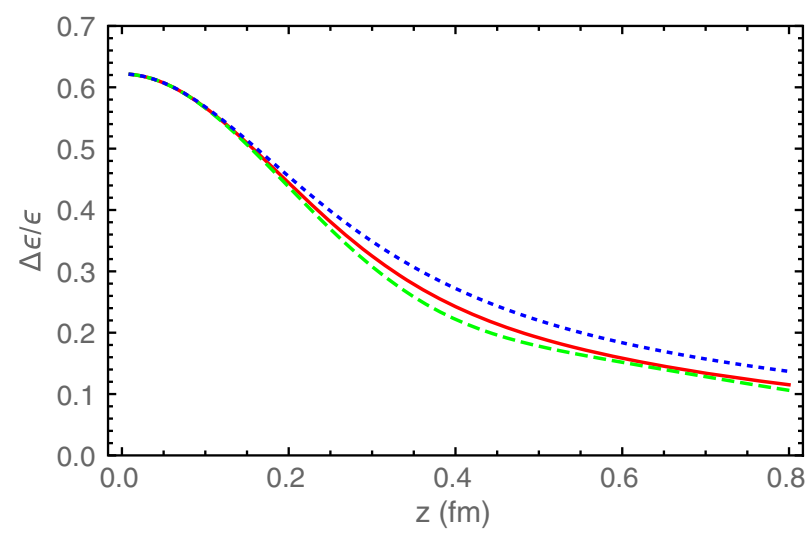

FIG. 2. The relative energy density fluctuation $\Delta \varepsilon(z) / \varepsilon_{0}$ for the three models considered here: the MV model is shown as a solid line (red), model 1 as a dashed line (green), and model 2 as a dotted line (blue). 
As a result of these corrections, Eq. (26) in MS is no longer valid.

Two of the figures contained in our manuscript are in need of correction. Figure 3 of MS is replaced with Fig. 1, where the solid (blue) line shows the new result, and the dashed (red) line shows the published incorrect result. The correction results in a reduction of the short-range energy density fluctuations by approximately $10 \%$, but leaves the long-distance tail of the energy density correlation function unchanged. Figure 5 of MS is replaced with Fig. 2.

We thank Tuomas Lappi and Soren Schlichting [1] for alerting us to the errors in our published manuscript.

[1] T. Lappi and S. Schlichting, arXiv:1708.08625. 